\title{
A TECHNIQUE FOR RECOVERING THE VERTICAL NUMBER DENSITY PROFILE OF ATMOSPHERIC GASES FROM PLANETARY OCCULTATION DATA
}

\author{
R. G. ROBLE \\ National Center for Atmospheric Research, ${ }^{*}$ Boulder, Colorado 80302, U.S.A \\ and \\ P. B. HAYS \\ Department of Aerospace Engineering, University of Michigan, \\ Ann Arbor, Michigan 48104, U.S.A
}

(Received 29 March 1972)

\begin{abstract}
The occultation technique of determining the properties of the atmosphere using absorption spectroscopy is examined. The intensity of a star, in certain atmospheric absorption bands, is monitored by a satellite tracking the star during occultation by the Earth's atmosphere. The intensity data in certain wavelength intervals, where absorption is attributed to a single species, are related to the tangential column number density of the absorbing species through Beer's law. The equation for the tangential column number density is the Abel integral equation which is inverted to obtain the number density profile of the absorbing species at the occultation tangent ray point. Two numerical schemes for inverting the Abel integral equation for signals of low intensity with statistical noise superimposed are presented; one for determining the number density profile of atmospheric species that decrease exponentially with height, and the second for determining the profile of constituents having a more complex vertical structure, such as ozone. The accuracy of retrieving the number density distribution from planetary occultation data is examined. A theoretical analysis of the errors in determining the number density from occultation data of very low signal intensity is also presented. The errors in retrieving the number density profile are related to the intensity of the source, the number of data points per scan, and the degree of data smoothing required before inversion. As a specific example, calculations are made of the errors in retrieving the molecular oxygen and ozone number density profiles from occultation intensity data in the Schumann-Runge continuum of molecular oxygen at $1450 \AA$ and the Hartley continuum of ozone at $2450 \AA$.
\end{abstract}

\section{INTRODUCTION}

Absorption spectroscopy is an important technique for determining the composition and number density profiles of constituents in the upper atmosphere (Watanabe, 1958; Hinteregger, 1962). Most of the measurements have been made from rockets monitoring the absorption of the solar u.v. flux in certain atmospheric bands (Johnson et al., 1951; Bryam et al., 1957; Kupperian et al., 1959; Jursa et al., 1963, 1965; Carver et al., 1964, 1966; Weeks and Smith, 1968; Opal and Moos, 1969; Wildman et al., 1969; Quessette, 1970; Brannon and Hoffman, 1971). Satellites have also been used to measure the intensity of certain u.v. sources (i.e., Sun, Moon, stars) during occultation of the source by the Earth's atmosphere (Fig. 1). The intensity data measured during occultation are then used to obtain information about the absorbing species in the upper atmosphere (Venkateswaran et al., 1961; Rawcliffe et al., 1963; Miller and Stewart, 1965; Thomas et al., 1965; Fesenkov, 1967; Thomas and Norton, 1967; Norton and Warnock, 1968; Stewart and Wildman, 1969; Hinteregger and Hall, 1969; Lockey et al., 1969; Link, 1969; Reid and Withbroe, 1970; May, 1971; Reid, 1971; Roble and Norton, 1972; Hays et al., 1972).

Basically, the occultation technique is similar to the classical technique of absorption spectroscopy. The Sun, Moon, or a star are used as a source of light and the satellite

- The National Center for Atmospheric Research is sponsored by the National Science Foundation. 


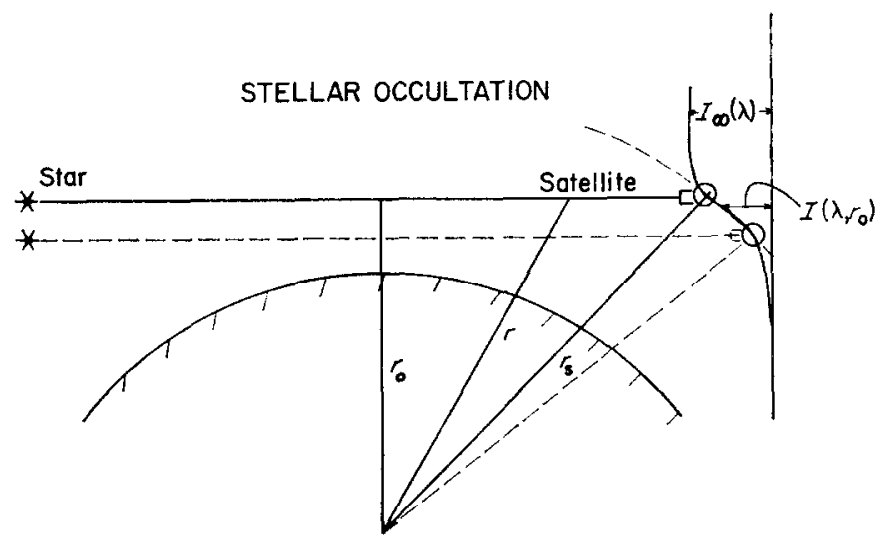

Fig. 1. Geometry OF STELLAR OCCULTATION. $I_{\infty}(\lambda)$ IS THE INTENSTTY OF THE UNATteNUATED STAR ABOVE THE ATMOSPHERE AND $I\left(\lambda, r_{0}\right)$ IS THE MEASURED INTENSITY OF THE STAR AT TANGENT RAY HEIGHT $r_{0}$.

photometers as a detector, with the atmosphere between acting as the absorption cell. As the satellite moves in its orbit, the source ultimately is occulted by the Earth. During the occultation process, the intervening atmosphere absorbs progressively more of the light due to the strong photo-absorption features of the atmospheric gases. The occultation intensity data can be related to the tangential column number density of the absorbing species if the absorption cross-sections are known. The equation for the tangential column number density of the absorbing species is the Abel integral equation which is easily inverted to give the number density profile at the occultation tangent point. The occultation technique works best for a point source, such as a star, where the vertical resolution at the tangent point is very sharp (Hays and Roble, 1968b). For a finite-size source, such as the Sun, the light rays emitted from the upper and lower limb can have tangent ray heights separated by about $26 \mathrm{~km}$ when observed from normal satellite altitudes. This complicates the problem of relating the transmitted solar intensity to the tangential column number density (May, 1971; Roble and Norton, 1972). However, once the tangential column number density is obtained, it again becomes easy to invert the Abel integral equation and obtain the local number density at the tangent ray point. For simplicity, we therefore assume that the light source is small, such as a star (Hays et al., 1972) or a small portion of the solar disk (Hinteregger and Hall, 1969; Reid and Withbroe, 1970; Reid, 1971).

In the present paper, we describe a technique for retrieving the number density profile of the absorbing species from occultation intensity data. The data-reduction procedure is similar to the method of computing the radial emission distribution of a cylindrical plasma source, as treated by the plasma physicists. The similarity exists because both techniques reduce to the problem of solving the Abel integral equation (Hays and Roble, 1968a, b; Barr, 1962).

In practice, the numerical solution of the Abel integral equation requires special handling because of its sensitivity to small random errors in the data. Therefore, we develop two separate techniques of numerically inverting the Abel integral equation to obtain the number density distribution of the absorbing species. The first utilizes an exponential approximation for atmospheric species which decrease nearly exponentially with altitude; the second, a quadratic approximation which works better for atmospheric species having a more complex vertical distribution such as atmospheric ozone (Hays et al., 
1972). The retrieval accuracy of both schemes is examined, and a theoretical analysis of errors is presented which enables us to determine the accuracy of the measurement in the presence of random statistical errors inherent in light sources of very low intensity. The inversion technique and analysis of errors, although examined with respect to molecular oxygen and ozone, are general and can be used to determine the accuracy of any occultation measurement if the signal level and data-acquisition rate are known.

\section{OCCULTATION TECHNIQUE}

The intensity of a star, as measured by a telescope aboard a satellite, during occultation of the star by the Earth's atmosphere (Fig. 1) is expressed as

$$
F^{*}\left(r_{0}\right)=\varphi \int_{0}^{\infty} T(\lambda) I\left(\lambda, r_{0}\right) \mathrm{d} \lambda
$$

where $r_{0}$ is the tangent ray height of the light ray passing tangentially to the Earth's surface, $\varphi=A Q_{\varepsilon} T_{0}, A$ is the aperture area of the telescope, $Q_{\varepsilon}$ is the quantum efficiency of the photo-multiplier, $T_{0}$ is the optical transmission of the system, $T(\lambda)$ is the broadening function or transmission of the dispersive element at wavelength $\lambda$ and $I\left(\lambda, r_{0}\right)$ is the intensity of the star at wavelength $\lambda$ and tangent ray height $r_{0}$.

The intensity of starlight passing through the Earth's upper atmosphere is determined by considering the combined attenuation of all of the absorbing species along the ray path (Beer's law).

$$
I\left(\lambda, r_{0}\right)=I_{\infty}(\lambda) \exp \left(-\sum_{i} \sigma_{i}(\lambda) N_{i}\left(r_{0}\right)\right)
$$

where $I_{\infty}(\lambda)$ is the stellar intensity above the atmosphere and $\sigma_{i}(\lambda)$ is the absorption crosssection of the $i$ th atmospheric species. In the case of a spherically stratified atmosphere, the tangential column number density $N_{i}\left(r_{0}\right)$ is

$$
N_{i}\left(r_{0}\right)=2 \int_{r_{0}}^{\infty} \frac{n_{i}(r) r \mathrm{~d} r}{\sqrt{r^{2}-r_{0}^{2}}}
$$

where $n_{i}(r)$ is the number density of the $i$ th absorbing species at a distance $r$ from the center of the Earth.

Hays and Roble (1968b) calculated the tangential u.v. transmission for the mesosphere and the lower thermosphere. They show that in certain specific wavelength intervals the u.v. starlight is absorbed by a single species. These include a broadband region near $1500 \AA$ where molecular oxygen acts as a single absorber in the lower thermosphere and also a region near $2500 \AA$ where ozone is a single absorber in the mesosphere. In both of these regions, Hays and Roble (1968b) show that refractive attenuation, Rayleigh scattering, and absorption by other minor atmospheric constituents are negligible over most of the altitude range where absorption occurs. In addition, they point out that only the relative intensity is important for the occultation measurement. The stellar occultation data are therefore normalized as

$$
F\left(r_{0}\right)=\frac{\int_{0}^{\infty} T(\lambda) I_{\infty}(\lambda) \exp \left(-\sigma(\lambda) N\left(r_{0}\right)\right) \mathrm{d} \lambda}{\int_{0}^{\infty} T(\lambda) I_{\infty}(\lambda) \mathrm{d} \lambda} .
$$

The interference filter used in the occultation measurements is selected so that $T(\lambda)$ includes only a wavelength region where absorption is caused by the single species of 
interest. By knowing the filter-broadening function, the stellar spectra above the atmosphere, and the absorption cross-section, the normalized signal is then only a function of the tangential column number density $F\left(r_{0}\right)=F\left(N\left(r_{0}\right)\right.$ ). The intensity data can be inverted to give the tangential column number density $N\left(r_{0}\right)=N\left(F\left(r_{0}\right)\right.$ ). Equation (3) for the tangential column number density is the Abel integral equation (c.f. Hays and Roble, 1968a, b) which is directly inverted to give the number density of the absorbing species at radius $r$

$$
n_{i}(r)=-\frac{1}{\pi} \int_{r}^{\infty} \frac{\left[\mathrm{d} N\left(r_{0}\right) / \mathrm{d} r_{0}\right] \mathrm{d} r_{0}}{\sqrt{r_{0}{ }^{2}-r^{2}}}
$$

In the next sections, we develop two separate numerical inversion schemes; one applicable for determining the number density profile of atmospheric species decreasing nearly exponentially with altitude, such as molecular oxygen; and the second for atmospheric species having a more complex number density profile, such as ozone.

\section{DATA REDUCTION}

(a) Exponential form. The intensity of a star measured by photometers aboard a satellite during occultation is generally weak and usually obtained in digital form (Hays et al., 1972). At low intensities, pulse-counting systems are used with a finite integration time between data points. Therefore, the intensity data are obtained at a series of discrete tangent ray heights $F\left(r_{0}\right)$. The normalized intensities are converted to the tangential column number density of the absorbing species along the ray path $N\left(r_{0}\right)$ as described in the previous section. Thus, a set of discrete values of the tangential column number density is obtained as a function of tangent ray height during occultation. We approximate the tangential column number density between data points in the interval $r_{i}<r<r_{i+1}$ by

$$
N(r)=\alpha_{i} \exp \left(-\beta_{i}\left(r-r_{i}\right)\right)
$$

where $r_{i}$ and $r_{i+1}$ are the $i$ and $i+1$ tangent ray heights of the data points, respectively. The coefficients $\alpha_{i}$ and $\beta_{i}$ are determined from a least squares fit to the data in the vicinity of the $i$ th data point by minimizing the variance of Equation (6) with the data and smoothing $M$ adjacent points (c.f. Roble and Norton, 1972).

$$
\delta_{i}=\sum_{j=1}^{K_{2}}\left(N_{i}-\alpha_{i} \exp \left(-\beta_{i}\left(r_{j}-r_{i}\right)\right)\right)^{2}
$$

where $K_{1}=i-M / 2, K_{2}=i+M / 2, N_{j}$ is the tangential column number density at the $j$ th data point. Then by differentiating Equation (6) and replacing the integral in Equation (5) by a finite sum, we obtain

$$
n\left(r_{l}\right)=\frac{1}{\pi} \sum_{i=l}^{\infty} \alpha_{i} \beta_{i} \int_{r_{i}}^{r_{i+1}} \frac{\exp \left(-\beta_{i}\left(r-r_{i}\right)\right)}{\sqrt{r^{2}-r_{l}^{2}}} \mathrm{~d} r .
$$

Expanding the denominator and integrating, we obtain

$$
\begin{aligned}
\left.n\left(r_{l}\right)=\frac{1}{\pi} \sum_{i=l}^{\infty} \frac{\alpha_{i} \beta_{i}}{\left(r_{i}\right.}+r_{l}\right)^{1 / 2} & \left(1+\frac{1}{2} \frac{\left(r_{i}-r_{l}\right)}{\left(r_{i}+r_{l}\right)}-\frac{1}{4 \beta_{i}} \frac{1}{\left(r_{i}+r_{l}\right)}\right)\left(\frac{\pi}{\beta_{i}}\right)^{1 / 2} \\
& \times\left[\operatorname{erf}\left(\beta_{i}\left(r_{i+1}-r_{l}\right)\right)^{1 / 2}-\operatorname{erf}\left(\beta_{i}\left(r_{i}-r_{l}\right)\right)^{1 / 2}\right] \exp \left(\beta_{i}\left(r_{i}-r_{l}\right)\right) \\
& \left.\quad+\frac{1}{2 \beta_{i}\left(r_{i}+r_{l}\right)}\left[\left(r_{i+1}-r_{l}\right)^{1 / 2} \exp \left(-\beta_{i}\left(r_{i+1}-r_{i}\right)\right)-\left(r_{i}-r_{l}\right)^{1 / 2}\right]\right\}
\end{aligned}
$$

where $\operatorname{erf}(x)$ is the error function of $x$. 
Thus, the number density of the absorbing species at radius $r_{l}$ is determined directly from Equation (9) using the calculated values of $\alpha_{i}$ and $\beta_{i}$.

(b) Quadratic form. When the absorbing constituent has a number density height profile which is not exponentially decreasing with altitude, but varies over the scan altitude, such as for ozone (Fig. 2), then the exponential scheme developed in the previous section may not be

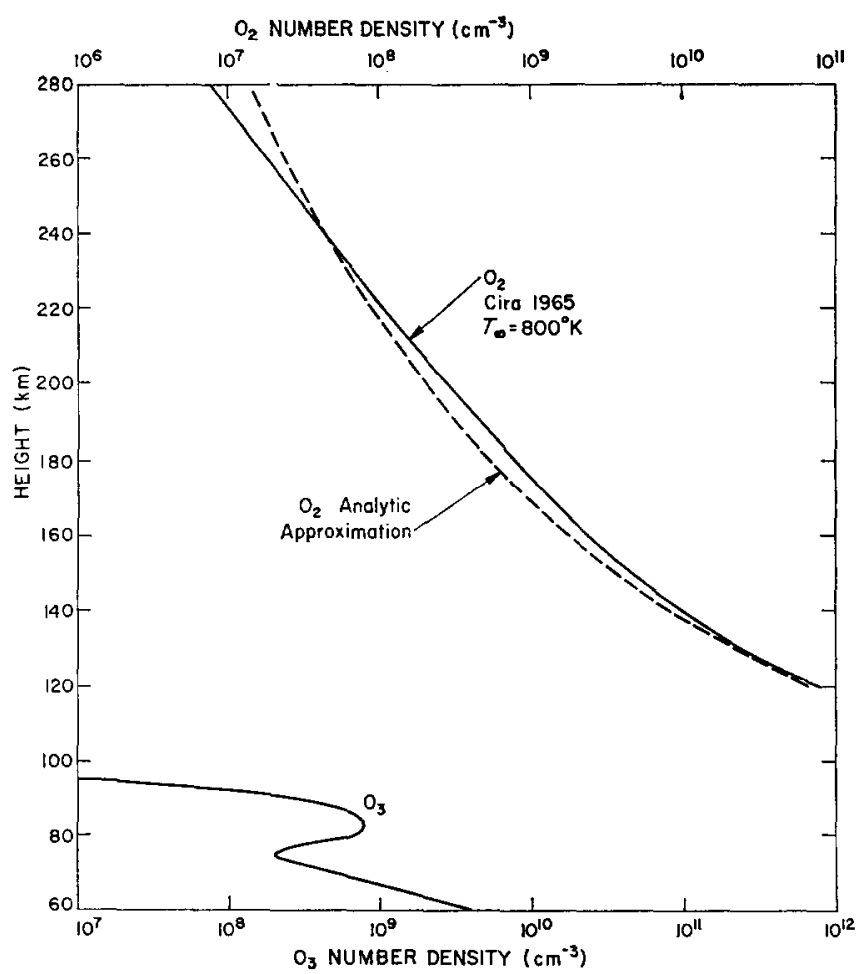

Fig. 2. The molecular oXygen AND Ozone Number Density as a Function Of height. THE SOLID CURVE FOR $\mathrm{O}_{2}$ IS THE NUMBER DENSITY DISTRIBUTION FROM THE MEAN CIRA 1965 MODEL ATMOSPHERE AND THE DASHED CURVE IS AN ANALYTIC APPROXIMATION TO THE DISTRIBUTION. THE $\mathrm{O}_{3}$ NUMBER DENSITY DISTRIBUTION IS AN ANALYTIC APPROXIMATION OF A NIGHT-TIME PROFILE AND IT IS GIVEN BY EQUATION (17).

best suited for the data-reduction process. In this case, we approximate the tangential column number density in the altitude region $z_{i}<z<z_{i+1}$ by the quadratic relationship

$$
N(z)=A z^{2}+B z+C
$$

where $z=r-r_{e}$ and $r_{e}$ is the radius of the Earth, $6371 \mathrm{~km}$.

The coefficients $A, B$ and $C$ are again determined by minimizing the variance of equation (10) with the data and smoothing $M$ adjacent points

$$
\delta_{i}=\sum_{j=K_{1}}^{K_{2}}\left(N_{j}-\left(A_{i} z_{j}{ }^{2}+B_{i} z_{j}+C_{i}\right)\right)^{2} .
$$


Minimizing Equation (11) and solving directly for $A_{i}, B_{i}$ and $C_{i}$ we obtain

$$
\begin{aligned}
& A_{i}=\frac{(M(\overline{N z})-(\bar{N})(\bar{z}))\left(M\left(\overline{z^{3}}\right)-(\bar{z})\left(\overline{z^{2}}\right)\right)-\left(\overline{M(\overline{N z})}-(\bar{N})\left(\overline{z^{2}}\right)\right)\left(M\left(\overline{z^{2}}\right)-(\bar{z})(\bar{z})\right)}{\left(M\left(\overline{z^{3}}\right)-\left(\overline{z^{2}}\right)(\bar{z})\right)\left(M\left(\overline{z^{3}}\right)-(\bar{z})\left(\overline{z^{2}}\right)\right)-\left(M\left(\overline{z^{4}}\right)-\left(\overline{z^{2}}\right)\left(\overline{z^{2}}\right)\right)\left(M\left(\overline{z^{2}}\right)-(\bar{z})(\bar{z})\right)} \\
& B_{i}=\frac{(M(\overline{N z})-(\bar{z})(\bar{N}))-\left(M\left(\overline{z^{3}}\right)-\left(\overline{z^{2}}\right)(\bar{z})\right) A_{i}}{\left(M\left(\overline{z^{2}}\right)-(\bar{z})(\bar{z})\right)} \\
& C_{i}=\frac{\left.(\bar{N})-\overline{\left(z^{2}\right.}\right) A_{i}-(\bar{z}) B_{i}}{M}
\end{aligned}
$$

where

$$
(T)=\sum_{j=K_{1}}^{K_{2}}()_{j}
$$

As in the previous section, by differentiating Equation (10) and replacing the main integral of equation (5) with a finite sum and then integrating between data points, we obtain

$$
\begin{aligned}
n\left(r_{l}\right)=-\frac{1}{\pi} \sum_{i=l}^{\infty}\left[2 A _ { i } \left(\left(r_{i+1}^{2}-r_{l}^{2}\right)^{1 / 2}-\right.\right. & \left.\left(r_{i}^{2}-r_{l}^{2}\right)^{1 / 2}\right) \\
& \left.+\left(B_{i}-2 A_{i} r_{\theta}\right) \log _{\theta} \frac{\left(r_{i+1}+\left(r_{i+1}^{2}-r_{l}^{2}\right)^{1 / 2}\right)}{\left(r_{i}+\left(r_{i}^{2}-r_{l}^{2}\right)^{1 / 2}\right)}\right] .
\end{aligned}
$$

Thus, the number density of the absorbing species as radius $r_{l}$ is determined from equation (13) using the coefficients $A_{i}$ and $B_{i}$ calculated for each data point.

\section{ATMOSPHERIC MODEL}

The molecular oxygen number density profile in the lower thermosphere, obtained from the mean CIRA 1965 model atmosphere, is shown in Fig. 2. For mathematical simplicity, we approximate this profile by an atmosphere with a constant scale height gradient (Nicolet, 1960),

$$
H=H_{s}+\beta\left(r-r_{s}\right)
$$

where $H_{s}$ is the scale height of the atmosphere at radius $r_{s}$, and $\beta$ is the vertical gradient of the scale height. The molecular oxygen number density is then represented as

$$
n(r)=n_{s}\left[\frac{H_{s}}{H_{s}+\beta\left(r-r_{s}\right)}\right]^{(2+\beta) / \beta}
$$

where $n_{s}=2 \times 10^{12} \mathrm{~cm}^{-3}, r_{s}=6471 \mathrm{~km}, H_{s}=5 \mathrm{~km}$, and $\beta=0.23$ to give the approximation to the CIRA 1965 molecular oxygen distribution shown in Fig. 2. The tangential column number density of molecular oxygen along the ray path is determined by inserting Equation (15) into Equation (3), expanding the denominator, and integrating to obtain

$$
\begin{aligned}
N\left(r_{0}\right)=\left(\frac{2}{r_{0}}\right)^{1 / 2} n_{s}\left[\frac{H_{s}}{H_{s}+\beta\left(r_{0}-r_{s}\right)}\right]^{1 / 8}\left\{\left[\frac{\beta}{H_{s}+\beta\left(r_{0}-r_{s}\right)}\right]^{-3 / 2} B\left(\frac{3}{2},\left(\frac{1}{\delta}-\frac{3}{2}\right)\right)\right. \\
\left.+r_{0}\left[\frac{\beta}{H_{s}+\beta\left(r_{0}-r_{s}\right)}\right]^{-1 / 2} B\left(\frac{1}{2},\left(\frac{1}{\delta}-\frac{1}{2}\right)\right)\right\}
\end{aligned}
$$

where $\delta=\beta /(1+\beta)$ and $B(x, y)$ is the Beta function, $B(x, y)=\gamma(x) \gamma(y) / \gamma(x+y)$ and $\gamma(x)$ is the Gamma function. The normalized intensity as a function of tangent ray height is calculated from Equation (2) and is shown in Fig. 3 for absorption at a single wavelength 
$1450 \AA$ in the Schumann-Runge continuum where the molecular oxygen absorption coefficient is approximately $2 \times 10^{-17} \mathrm{~cm}^{2}$.

Hays et al. (1972) presented a typical night-time ozone distribution determined from occultation measurements made by the Orbiting Astronomical Observatory (OAO-2). The

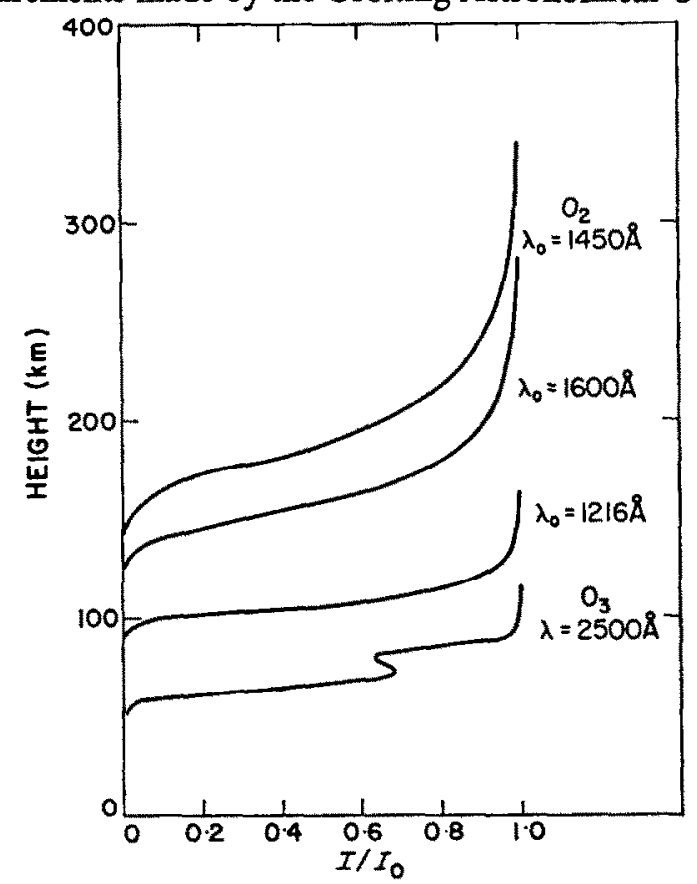

Fig. 3. ThB normalized tangential transmisston of tHB EARTH's atMosphere at Various WAVELENGTHS AS A FUNCTION OF HETGHT.

ozone number density profile given in their paper can be reasonably represented as a sum of exponential and Gaussian functions

$$
n(r)=n_{b} \exp \left(-\left(r-r_{b}\right) / H_{b}\right)+n_{a} \exp \left(-\left(\left(r-r_{c}\right) / H_{b}\right)^{2}\right)
$$

where $n_{b}=5 \times 10^{10} \mathrm{~cm}^{-3}, r_{b}=50 \mathrm{~km}, H_{b}=4.34 \mathrm{~km}, n_{c}=8 \times 10^{8} \mathrm{~cm}^{-3}, r_{c}=83 \mathrm{~km}$, and $H_{c}=5 \mathrm{~km}$. The ozone number density distribution obtained from equation (17) is shown in Fig. 2.

The coefficients used in this study give an ozone number density slightly higher than those for a typical night-time ozone distribution determined by Hays et al. (1972). This difference, however, is not important because the profiles are similar and the main features of the ozone analysis are preserved in either case.

The total columnar number density along a ray path is obtained by inserting equation (17) into equation (3), expanding the denominator, and integrating to give

$$
\begin{aligned}
& N\left(r_{0}\right)=2 n_{b} \exp \left(r_{b} / H_{b}\right) r_{0} K_{1}\left(r_{0} / H_{b}\right)+2 n_{c} \exp \left(-\left(\left(r_{0}-r_{c}\right) / H_{c}\right)^{2}\right) \\
& \times\left(2 r_{0}\right)^{-1 / 2}\left\{\left(\frac{2}{H_{c}^{2}}\right)^{-3 / 4} \gamma\left(\frac{3}{2}\right)\right. \exp \left(\left(r_{0}-r_{c}\right)^{2} /\left(2 H_{c}^{3}\right)\right) \\
& \times D_{-3 / 2}\left((2)^{1 / 2} H_{c}^{-1}\left(r_{0}-r_{c}\right)\right)+r_{0}\left(\frac{2}{H_{c}^{2}}\right)^{-1 / 4} \gamma\left(\frac{1}{2}\right) \\
&\left.\times \exp \left(\left(r_{0}-r_{c}\right)^{2} /\left(2 H_{c}^{2}\right)\right) D_{-1 / 2}\left((2)^{1 / 2} H_{c}^{-1}\left(r_{0}-r_{c}\right)\right)\right\}
\end{aligned}
$$


where $K_{1}(x)$ is the modified Bessel function of the first kind, $\gamma(x)$ is the Gamma function, and $D_{-v}(x)$ is the parabolic cylinder function. The normalized intensity as a function of tangent ray height is obtained from Equation (18) and Equation (2), and is shown in Fig. 3 for absorption at a single wavelength in the Hartley continuum of ozone at $2500 \AA$. At this wavelength, ozone has an absorption cross-section of about $10^{-17} \mathrm{~cm}^{-2}$.

\section{SAMPLING ERROR}

The accuracy in retrieving the number density profile with the data-reduction scheme depends upon (a) the data-acquisition rate or number of data points per scan, (b) the statistical noise inherent in a signal of low intensity, (c) the smoothing parameter $M$ used in the inversion process, (d) knowledge of the satellite position during occultation, and (e) departures from spherical symmetry. The first three items are examined in this paper. Item (d) depends on the satellite tracking network and the degree of accuracy of the orbital

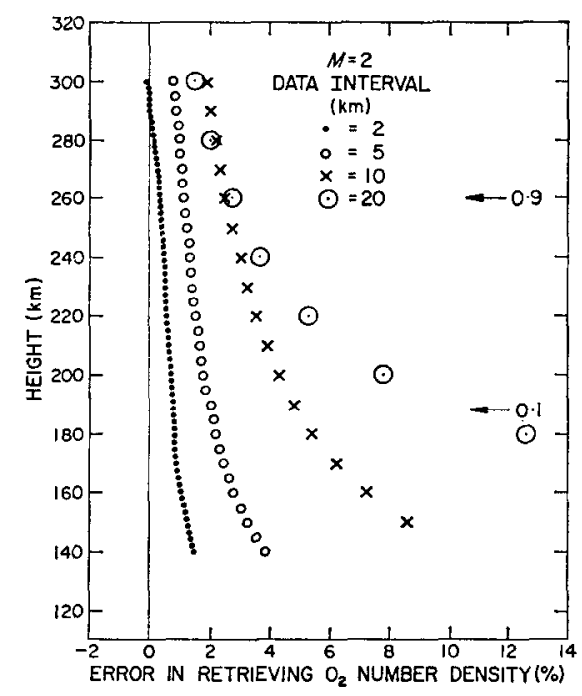

FIG. 4. THE ERROR IN RETRIEVING THE ANALYTIC MOLECULAR OXYGEN NUMBER DENSITY AS A FUNCTION OF HEIGHT FOR VARIOUS DATA-ACQUISITION INTERVALS.

elements. The intensity of the star is measured as a function of time, but by knowing the star's position and the satellite orbital elements, we can relate the intensity to the tangent ray height of the occulting star. Thus, errors in satellite time and position appear as errors in the tangent ray height and will not be discussed in this paper. The last item may be important for certain atmospheric species which change rapidly with time, as perhaps during sunrise or sunset; however, in this analysis, spherical symmetry is assumed.

The error in retrieving the molccular oxygen number density profile using the exponential data-reduction scheme is plotted as a function of height in Fig. 4 for various data-acquisition rates. The tangential column number density for the analytic molecular oxygen distribution is calculated at the various altitudes using Equation (16). These data are inverted using the exponential data-inversion scheme, and the retrieved molecular oxygen number density is compared to the number density given by the analytic model. The errors in retrieving the number density are then plotted in Fig. 4. The results show that the errors are smallest when a large number of data points per occultation scan are used in the 
inversion process. The smoothing parameter $M$ is also a factor in the retrieval accuracy. The smoothing parameter is primarily used to smooth the analytic representation of the tangential column number density through noisy data points and its importance will be evident in the next section. But for noise-free data, an increase in the smoothing parameter decreases the retrieval accuracy for a number density profile having a slight curvature.

At high altitudes, the retrieval accuracy would decrease due to the practical requirement of truncating the inversion integral at an upper boundary. It is difficult to convert the normalized intensity to tangential column number density for the upper part of the scan where the normalized intensity approaches $1 \cdot 0$, especially in the presence of statistical noise.

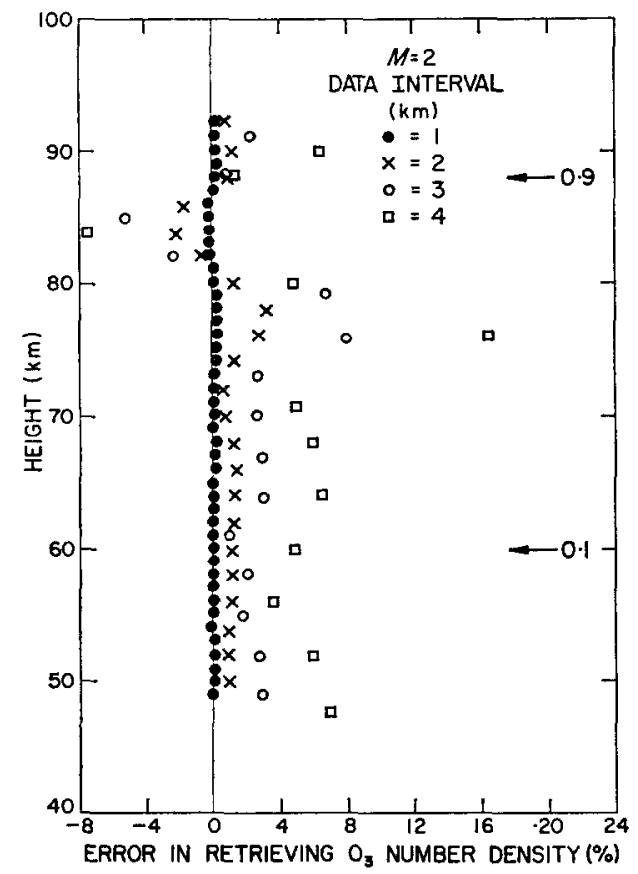

Fig. 5. THE ERROR IN RETRIEVING THE ANALYTIC OZONE NUMBER DENSITY AS A FUNCTION OF HEIGHT FOR VARIOUS DATA-ACQUISITION INTERVALS.

To reduce this error and extend the upper limit of the occultation scan, it is convenient to utilize a model atmosphere and calculate the tangential column number beyond the uppermost data point for use in the inversion integral.

The error in retrieving the model ozone number density profile using the analytic expression for the tangential column number density given by Equation (18) and also using the quadratic data-reduction scheme is shown in Fig. 5. The retrieval is good for a high data-acquisition rate, but as the data-acquisition rate decreases, the accuracy in defining the vertical structure and in particular the ozone bulge near $83 \mathrm{~km}$ also decreases. The lowest data-acquisition rate considered in Fig. 5 shows considerable error in retrieving the ozone bulge. These results were obtained using a smoothing parameter of $M=2$. As the smoothing parameter is increased, the accuracy of retrieving the vertical structure depends critically on the data-acquisition rate. For a particular application, there is a tradeoff between the data-acquisition rate and the smoothing parameter.

For simplicity, the results presented here assume absorption at a single wavelength. 
However, the technique which has been developed is general and can be applied to any broadband filter by including a wavelength integration.

\section{THEORETICAL ANALYSIS OF STATISTICAL ERRORS}

For occultation scans of very low intensity, the statistical noise which is superimposed upon the basic signal intensity becomes an appreciable part of the signal. The errors in retrieving the number density profile of the absorbing species from occultation data having random statistical noise are considered in this section.

(a) Molecular oxygen. The solid curve in Fig. 6 shows the calculated normalized intensity variation of a star during occultation using Equations (16) and (2). The peak signal

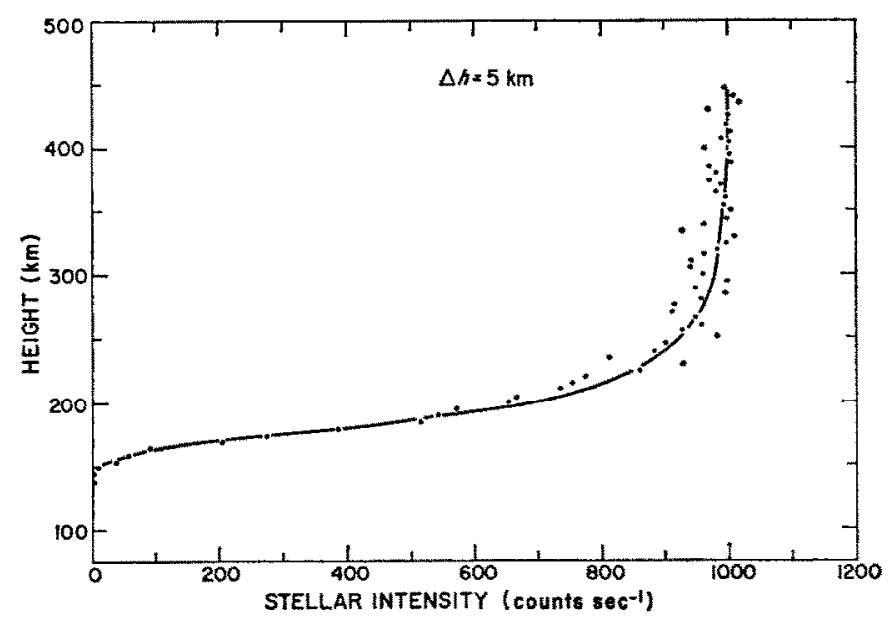

FIG. 6. AN OCCULTATION SCAN IN THE SCHUMANN-RUNGe CONTTNUUM OF MOLECULAR OXYGEN AT $1450 \AA$ WITH RANDOMLY SCATTERED STATISTICAL NOISE SUPERIMPOSED. THE STANDARD DEVIATTON OF THE NOISE IS PROPORTIONAL TO THE SQUARE ROOT OF THE BASIC SIGNAL LEVEL.

level in this example is approximately 1000 counts $\mathrm{sec}^{-1}$ and the calculations are made at a single wavelength of $1450 \AA$. Also shown in Fig. 6 is the basic scan with a superimposed Poisson noise distribution having a standard deviation proportional to the square root of the signal level. The molecular oxygen number density profiles shown in Fig. 7 for the two scans given in Fig. 6 have been retrieved using the data-reduction scheme described in the previous sections. Other statistical noise distributions yield an ensemble of retrieved molecular oxygen number density profiles which can be statistically analyzed.

If we assume that the tangential column number density is $N=N_{0}+\Delta N$ where $N_{0}$ is the basic tangential column number density without statistical noise and $\Delta N$ is the tangential column number density error due to the statistical noise, then in the approximation given by Equation (6) we expand $\alpha$ and $\beta$ as

$$
\begin{aligned}
& \alpha=\alpha_{0}+\Delta \alpha \\
& \beta=\beta_{0}+\Delta \beta .
\end{aligned}
$$

Inserting these relationships into Equation (7), expanding the exponential, and subtracting the basic unscattered state, we obtain

$$
\delta_{i}^{*}=\sum_{j=K_{i}}^{K_{2}}\left[\Delta N_{j}-\Delta \alpha_{i} \exp \left(-\beta_{0 i}\left(z_{j}-z_{i}\right)\right)+\alpha_{0 i} \Delta \beta_{i}\left(z_{j}-z_{i}\right) \exp \left(-\beta_{0 i}\left(z_{j}-z_{i}\right)\right)\right]^{2} .
$$




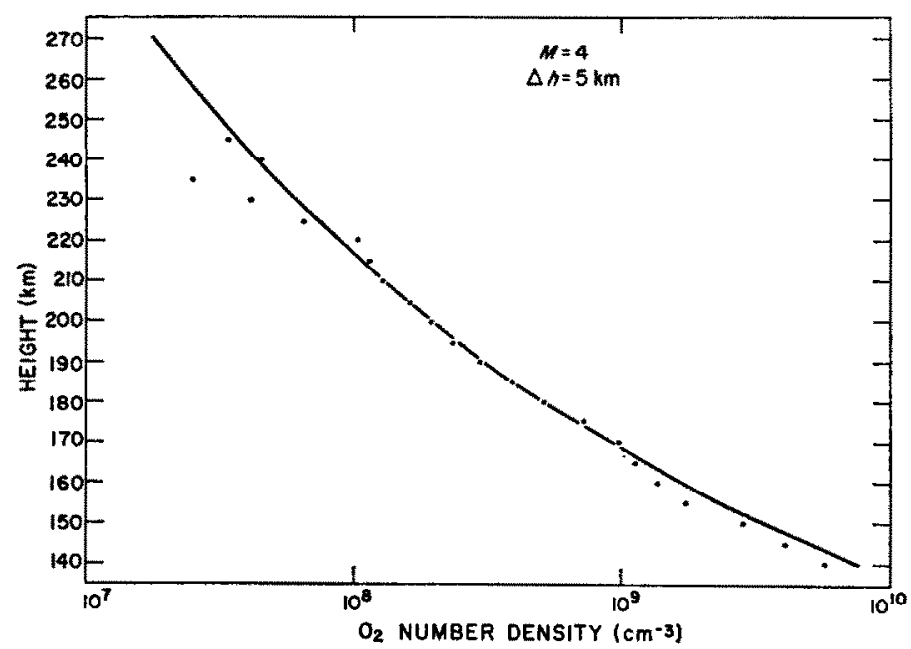

FIG. 7. THE RETRIEVED MOLECULAR OXYGEN NUMBER DENSITY PROFILE FOR THE OCCULTATION SCAN WTHH RANDOMLY SCATTERED NOISE SHOWN IN FIG. 6. THE SOLID CURVE GIVES THE ANALYTIC MOLECULAR OXYGEN NUMBER DENSTTY DISTRIBUTION.

The coefficient errors $\Delta \alpha_{i}$ and $\Delta \beta_{i}$ are calculated in the least squares sense from the relationships

and are given in the Appendix.

$$
\frac{\partial \delta_{i}^{*}}{\partial \Delta \alpha_{i}}=\frac{\partial \delta_{i}^{*}}{\partial \Delta \beta_{i}}=0
$$

The intensity of the star, considering single wavelength absorption, is calculated from Beer's law

$$
F_{0}=F\left(\lambda, r_{0}\right)=F_{\infty}(\lambda) \exp \left(-\sigma(\lambda) N\left(r_{0}\right)\right)
$$

where $F_{\infty}(\lambda)$ is the signal measured above the atmosphere in counts $\sec ^{-1}$. The linear variation in tangential column number density due to a signal with statistical noise $F=$ $F_{0}+\Delta F$ is obtained by introducing the value of $N$ into Equation (22) and expanding the exponential to obtain

$$
\Delta N=-\frac{1}{\sigma(\lambda)} \frac{\Delta F}{F\left(\lambda, r_{0}\right)} .
$$

If a Poisson random noise with a standard deviation for $\Delta F$ proportional to the square root of the basic signal $\left(F_{0}\right)^{1 / 2}$ is introduced into the expressions for the coefficients $\Delta \alpha_{i}$ and $\Delta \beta_{i}$, the mean square errors for the coefficients $\left\langle\overline{\Delta \alpha_{i}}\right\rangle$ and $\left\langle\overline{\Delta \beta_{i}}\right\rangle$ are determined using the propagation of statistical errors theorem and they are presented in the Appendix.

The errors in the number density of the absorbing species at the tangent ray point are determined from the integral

$$
\Delta n\left(r_{l}\right)=-\frac{1}{\pi} \int_{r_{l}}^{\infty} \frac{[\mathrm{d} \Delta N / \mathrm{d} r] \mathrm{d} r}{\sqrt{r^{2}-r_{l}^{2}}} .
$$

The tangential column number density of the absorbing species is determined by inserting Equation (19) into Equation (6). The noise in the number density of the absorbing 
species at the tangent ray point is obtained by expanding the exponential, subtracting the basic state, and differentiating the tangential column number density error $\Delta N$ to give

$$
\begin{aligned}
\Delta n\left(r_{l}\right)=\frac{1}{\pi} \sum_{i=l}^{\infty}\left(\beta_{0 i} \Delta \alpha_{i}+\alpha_{0 i} \Delta \beta_{i}\right) & \int_{r_{i}}^{r_{i+1}} \frac{\exp \left(-\beta_{0 i}\left(r-r_{i}\right)\right)}{\sqrt{r^{2}-r_{l}^{2}}} \mathrm{~d} r \\
& -\frac{1}{\pi} \sum_{i=l}^{\infty} \alpha_{0 i} \beta_{0 i} \Delta \beta_{i} \int_{r i}^{r_{i+1}} \frac{\left(r-r_{i}\right) \exp \left(-\beta_{0 i}\left(r-r_{i}\right)\right)}{\sqrt{r^{2}-r^{2}}} \mathrm{~d} r
\end{aligned}
$$

Integrating, we obtain

$$
\Delta n\left(r_{l}\right)=\frac{1}{\pi} \sum_{i=l}^{\infty}\left(R_{i} \Delta \alpha_{i}+S_{i} \Delta \beta_{i}\right)
$$

where $R_{i}$ and $S_{i}$ are coefficients given in the Appendix. The standard deviation of the number density error at the tangent ray point due to statistical noise is calculated using the propagation of statistical errors theorem

where

$$
\left\langle\Delta n\left(r_{l}\right)\right\rangle=T_{l} F_{\infty}^{-1 / 2}
$$

$$
T_{l}=\frac{1}{\pi}\left[\sum_{i=l}^{\infty}\left(R_{i} X_{i}+S_{i} Y_{i}\right)^{2}\right]^{1 / 2}
$$

and $X_{i}$ and $Y_{i}$ are given in the Appendix.

(b) Ozone. The errors in the ozone number density caused by statistical noise superimposed upon the basic signal are determined from the quadratic data-reduction scheme. The signal intensity variations are related to the tangential column number density variations through Equation (23) and the number density error of ozone at the tangent ray point is obtained from Equation (24). The tangential column number density with superimposed variations caused by statistical noise is $N=N_{0}+\Delta N$. The coefficients in Equation (10) are assumed to have a variation $A_{i}=A_{0 i}+\Delta A_{i}, B_{i}=B_{0 i}+\Delta B_{i}$, and $C_{i}=C_{0 i}+\Delta C_{i}$. Introducing these variations into Equation (11) and subtracting the basic state, we have

$$
\delta_{i}^{*}=\sum_{j=K 1}^{K_{2}}\left(\Delta N_{j}-\Delta A_{i} z_{j}{ }^{2}-\Delta B_{i} z_{j}-\Delta C_{i}\right)^{2} .
$$

Solving for the coefficients $\Delta A_{i}, \Delta B_{i}$ and $\Delta C_{i}$, we obtain relationships similar to Equation (12) with the exception that $N_{j}$ is replaced by $\Delta N_{j}$

$$
\begin{aligned}
\Delta A_{i} & =\sum_{j=K_{1}}^{K_{2}} Q_{j} \Delta N_{j} \\
\Delta B_{i} & =\sum_{j=K_{1}}^{K_{2}} Q_{i}^{*} \Delta N_{j}
\end{aligned}
$$

where $Q_{j}$ and $Q_{j}{ }^{*}$ are given in the Appendix. The number density error at the tangent ray point is obtained by inserting Equation (29) into Equation (24) and integrating to give

where

$$
\Delta n\left(r_{l}\right)=-\frac{1}{\pi} \sum_{i=l}^{\infty}\left[\sum_{j=K_{1}}^{K_{i}}\left(\left(2 W_{1 i}-2 r_{e} W_{2 i}\right) Q_{j}+W_{2 i} Q_{j}^{*}\right) \cdot \frac{\Delta F_{j}}{\sigma F_{0 j}}\right]
$$

$$
\begin{aligned}
& W_{1 i}=\left(r_{i+1}^{2}-r_{l}^{2}\right)^{1 / 2}-\left(r_{i}^{2}-r_{l}^{2}\right)^{1 / 2} \\
& W_{2 i}=\log _{e}\left(\frac{r_{i+1}+\left(r_{i+1}^{2}-r_{l}^{2}\right)^{1 / 2}}{r_{i}+\left(r_{i}^{2}-r_{l}^{2}\right)^{1 / 2}}\right)
\end{aligned}
$$


The standard deviation of the number density error at the tangent ray point is calculated using the propagation of statistical errors theorem and assuming that the standard deviation of the noise is proportional to the square root of the basic signal

where

$$
\left\langle\Delta n\left(r_{l}\right)\right\rangle=E_{l} \cdot F_{\infty}^{-1 / 2}
$$

$$
E_{l}=\frac{1}{\pi}\left\{\sum_{i=l}^{\infty} \sum_{j=K_{1}}^{K_{2}}\left[\left[\left(2 W_{1 i}-2 r_{e} W_{2 i}\right) Q_{j}+W_{2 i} Q_{j}^{*}\right] \frac{1}{\sigma}\left(\frac{F_{\infty}}{F_{0 j}}\right)^{1 / 2}\right]^{2}\right\}^{1 / 2} .
$$

\section{ANALYSIS OF STATISTICAL ERRORS}

The error in retrieving the molecular oxygen number density from occultation scans having a random statistical noise superimposed upon the basic signal is defined as

$$
e\left(r_{l}\right)=\frac{\left\langle\Delta n\left(r_{l}\right)\right\rangle}{n\left(r_{l}\right)}
$$

The numerator is the standard deviation of the retrieved number density, given by equation (27) for molecular oxygen and equation (31) for ozone, and $n\left(r_{l}\right)$ is the number density at radius $r_{i}$. The standard deviation of the retrieved number density is inversely proportional to the square root of the unattenuated signal level for both molecular oxygen and ozone. Therefore, in Fig. $8 T_{l}\left(n\left(r_{l}\right)\right)^{-1}$ is plotted as a function of altitude for various smoothing parameters $M$. The error in retrieving the molecular oxygen number density for

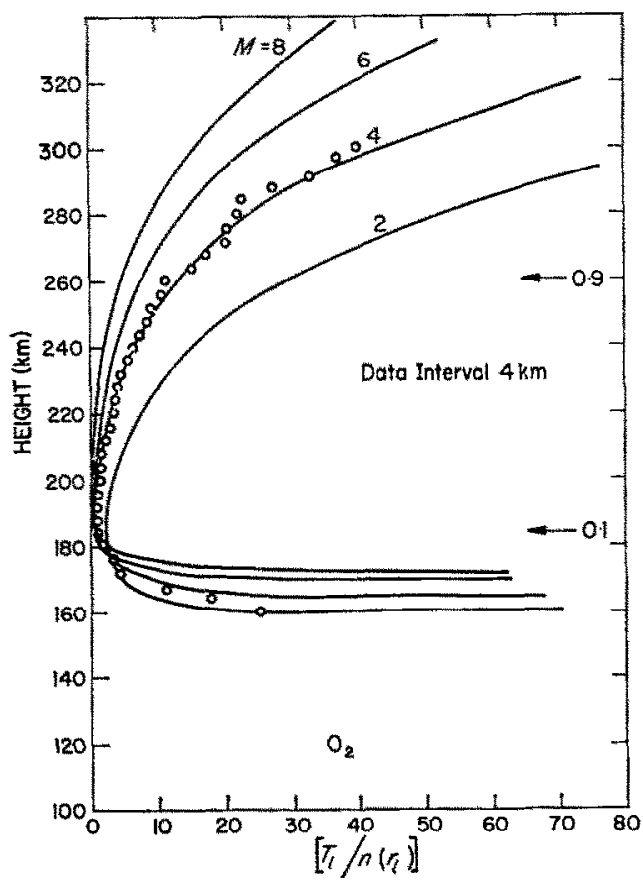

FIG. 8. THEORETICAL ANALYSIS OF ERRORS IN RETRIEVING THE MOLECULAR OXYGEN NUMPER DENSITY FROM OCCULTATION SCANS WTH RANDUM STATISTICAL NOISE SUPERIMPOSED. THE RETRIEVAL ERROR IS OBTAINED BY DIVIDNG THE PARAMETER $T_{l}\left(n\left(r_{l}\right)^{-1}\right.$ BY $F_{\infty}^{1 / 2}$, THE SQUARE ROOT OF THE UNATTENUATED BASIC SIGNAL LEVEL. THE CIRCLES REPRESENT THE RESULTS OF A NUMERICAL ANALYSIS OF ERRORS FOR $M=4$. 
any signal level is obtained by dividing the parameter $T_{l}\left(n\left(r_{l}\right)\right)^{-1}$ by $F_{\infty}^{1 / 2}$. An increase in the smoothing parameter $M$ decreases the error in retrieving the molecular oxygen number density. However, as discussed earlier, the accuracy of retrieving the basic molecular oxygen number density profile from unscattered occultation scans decreases as the smoothing parameter $M$ increases. Therefore, there exists a value of $M$ which gives a minimum in the overall error in retrieving the molecular oxygen number density profile at a given dataacquisition interval. The results of a numerical analysis of errors are shown in Fig. 8 for 100 occultation scans with different randomly scattered noise distributions. There is general

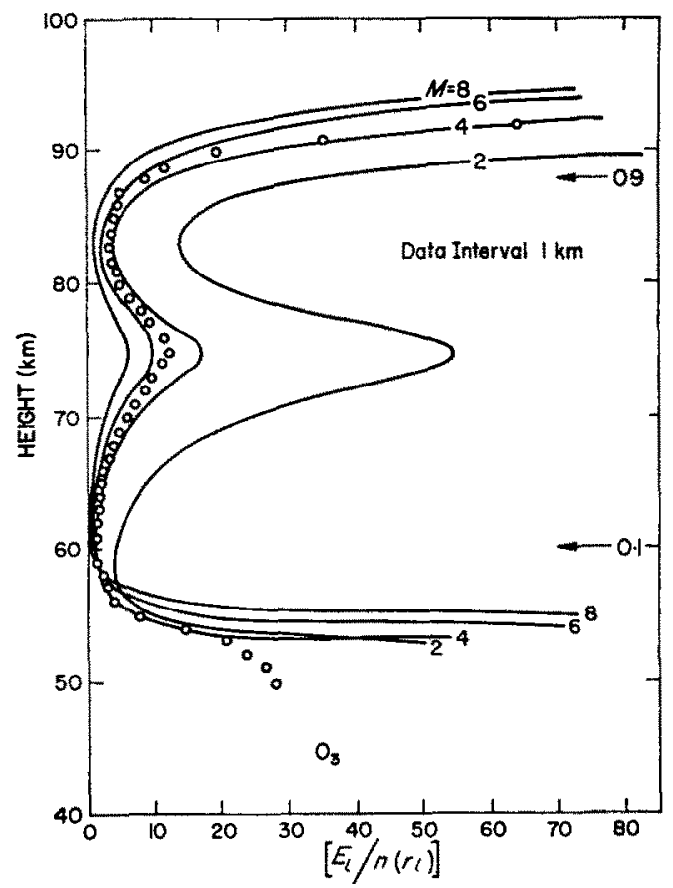

FIG. 9. THEORETICAL ANALYSTS OF ERRORS IN RETRIEVING THE OZONE NUMBER DENSITY FROM OCCULTATION SCANS WITH RANDOM STATISTICAL NOISE SUPERIMPOSED. THE RETRIEVAL ERKOK IS OBTAINED BY DIVIDING THE PARAMETER $E_{l}\left(n\left(r_{l}\right)\right)^{-1}$ BY $F_{\infty}^{1 / 2}$, THE SQUARE ROOT OF THE UNATTENUATED BASIC SIGNAL LEVEL. THE CIRCLES REPRESENT THE RESULTS OF A NUMERICAL ANALYSIS OF ERRORS FOR $M=4$.

agreement between the results of the theoretical and numerical analysis of errors. Also shown in the figure are arrows indicating the altitudes of the 0.1 and 0.9 normalized intensity points. The retrieval of the molecular oxygen number density is best for data lying between these normalized intensity ratios.

The standard deviation of the retrieved ozone number density is also inversely proportional to the square root of the unattenuated signal level. Therefore, in Fig. 9 the parameter $E_{l}\left(n\left(r_{l}\right)\right)^{-1}$ is plotted as a function of height for various smoothing parameters. In general, the error in retrieving the ozone bulge and the number density below about $70 \mathrm{~km}$ is low. However, in the region of the ozone minimum near $75 \mathrm{~km}$ the retrieval error increases. The data-acquisition interval in this case is $1 \mathrm{~km}$ and an increase in the smoothing parameter $M$ decreases the error in retrieving the ozone number density. As the dataacquisition interval increases, the error in retrieving the ozone number density increases. 
For a given data-acquisition interval, there also is a value of the smoothing parameter $M$ which gives a minimum in the overall error in retrieving the ozone number density. The circles in Fig. 9 again are the results of a numerical analysis of the errors for 100 ozone occultation scans with different randomly scattered noise distributions. The general agreement between the theoretical and numerical analysis of errors enables us to theoretically calculate the error coefficients and thus determine accuracy of an occultation scan for any signal intensity.

\section{DISCUSSION}

We have presented a numerical technique for retrieving the number density profile of absorbing atmospheric gases from planetary occultation data having random statistical noise superimposed upon the basic signal. Because of the sensitivity of the Abel integral equation to random noise, some smoothing is required before evaluating the inversion integral. An exponential technique was developed for atmospheric species whose number density profile decreases exponentially with height, whereas the quadratic scheme works better for atmospheric species having a more complex vertical profile. These techniques have been used to reduce the OAO-2 stellar occultation data and obtain the night-time molecular oxygen and ozone distribution in the upper atmosphere (Hays et al., 1972). With a modification to account for the finite size of the solar disk, these techniques have also been used to reduce the SOLRAD-8 solar occultation data (Roble and Norton, 1972). The retrieval errors are determined by knowing the approximate signal level and acquisition rate of the occultation data. For each specific case, there is a minimum in the retrieval error determined by the degree of smoothing for the appropriate data-acquisition rate and basic signal level. Generally, the best accuracy is achieved between the normalized intensity limits of $0 \cdot 1-0.9$ where the derivative in the numerator of the Abel integral equation changes rapidly. Figure 2 shows that by selecting the appropriate spectrally isolated wavelength interval, the number density distribution at practically any altitude may be obtained in the region where the tangential column number density is approximately equal to the inverse of the effective cross-section.

The analysis was performed for single wavelength absorption, whereas measurements are made for some finite passband determined by the characteristics of the monitoring spectrometer or photometer. In this case, an additional integration over wavelength is required in the analysis, but it does not fundamentally alter the results as long as the absorption process is spectrally isolated. Actually, a wavelength interval selected to cover a continuum region where the absorption cross-section is smoothly increasing or decreasing allows deepest penetration into the atmosphere. The altitude range of the occultation data is greater in this case than for the case of a constant cross-section over the selected wavelength interval.

Acknowledgments-We are grateful for the assistance of Messrs. K. Hansen, J. Hastings and M. Graves who provided the numerical calculations and plotting. A. Lundberg typed the manuscript.

\section{REFERENCES}

BARR, W. L. (1962). Method for computing the radial distribution of emitters in a cylindrical source. J. opt. Soc. Amer. 52, 885.

Brannon, P. J. and Hoffman, J. M. (1971). Molecular oxygen density measurements from 80 to 140 kilometers. J. geophys. Res. 76, 4630.

Bryam, E. T., Chubb, T. A. and Frigdman, H. (1957). The dissociation of molecular oxygen at high altitudes, in Threshold of Space, 211 pp. Pergamon Press, London. 
CARver, J. H., MitchelX, P. and MURRAY, E. L. (1964). Molecular oxygen density and Lyman- $\alpha$ absorption in the upper atmosphere. J. geophys. Res, 69, 3755,

CARver, J. H., Horton, B. H. and Burger, F. G. (1966). Nocturnal ozone distribution in the upper atmosphere. J. geophys. Res. 74, 6873.

CIRA (1965). COSPAR International Reference Atmosphere, 312 pp. North-Holland, Amsterdam.

FEsENKov, V. G. (1967). A satellite technique for sounding the optical properties of the atmosphere. Soviet Astronomy-AJ, 11, 1.

HAYS, P. B. and Roble, R. G. (1968a). Atmospheric properties from the inversion of planetary occultation data. Planet. Space Sci. 16, 1197.

HAYs, P. B. and RoBLE, R. G. (1968b). Stellar spectra and atmospheric composition. J. atmos. Sci. 25, 1141 .

Hays, P. B., Roble, R. G. and ShaH, A. N. (1972). Terrestrial atmospheric composition from stellar occultations. Science 176, 793.

HintrREGGER, H. E. (1962). Absorption spectrometric analysis of the upper atmosphere in the EUV region. J. atmos. Sci. 19, 351 .

HINTEREGGeR, H. E. and HALL, L. A. (1969). Thermospheric densities and temperatures from EUV absorption measurements by OSO-III. Space Res. IX, 519. North-Holland.

Johnson, F. S., Purcell, J. D. and Tousey, R. (1951). Measurements of the vertical distribution of atmospheric ozone from rockets. J. geophys, Res. 56, 583.

JURSA, A. S., NAKAmURA, M. and TANAKA, Y. (1963). Molecular oxygen distribution in the upper atmosphere. J. geophys. Res. 68, 6145.

JuRsa, A. S., Nakamura, M. and Tanaka, Y. (1965). Molecular oxygen distribution in the upper atmosphere-II. J. geophys. Res. 70, 2699.

Kupperian, J. E., Bryam, E. T. and Friedman, H. (1969). Molecular oxygen densities in the mesosphere at Fort Churchill. J. atmos terr. Phys. 16, 174.

LiNK, F. (1969). Eclipse Phenomena in Astronomy, 268 pp. Springer-Verlag, New York.

LOCKEY, G. W. A., HORTON, B. H. and ROFE, B. (1969). Satellite measurement of upper atmospheric molecular oxygen density. Nature Lond. 223, 387.

MAY, B. R. (1971). A method of determining the density of thermospheric gases from measurements of solar ultra-violet light absorption at grazing-ray and near-vertical incidence. Planet. Space Sci. 19, 27.

MILLRR, D. E. and StewarT, K. H. (1965). Observations of atmospheric ozone from an artificial earth satellite. Proc. R. Soc. A288, 540.

Nicolet, M. (1960). The properties and constitution of the upper atmosphere, in Physics of the Upper Atmosphere (Ed. J. A. Ratcliffe), pp. 577. Academic Press, New York.

NORTON, R. B. and WARNOCK, J. M. (1968). Scasonal variation of molccular oxygen near 100 kilometers. J. geophys. Res. 73, 5798.

OpAL, C. B. and Moos, H. W. (1969). Night-time molecular oxygen densities in the 100-130 km region from Schumann-Runge absorption. J. geophys. Res. 74, 2398.

QUESSETTE, J. A. (1970). On the measurement of molecular oxygen concentration by absorption spectroscopy. J. geophys. Res. 75, 839.

RawCliffe, R. D., Meloy, G. E., Friedman, R. M. and Rogers, E. H. (1963). Measurement of yertical distribution of ozone from a polar orbiting satellite. J. geophys. Res. 68,6425 .

REID, R. H. G. (1971). Number densities of atomic oxygen and molecular nitrogen in the thermosphere. Planet. Space Sci. 19, 801.

REID, R. H. G. and WITHBroe, G. L. (1970). The density and vibrational distribution of molecular oxygen in the lower thermosphere. Planet. Space Sct. 18, 1255 .

RoBle, R. G. and Norton, R. B. (1972). Thermospheric molecular oxygen from solar u-v occultation data. Submitted to J. geophys. Res. 77 .

Stewart, K. H. and WrLdman, P. J. L. (1967). Preliminary results of molecular oxygen observations from the Ariel III satellite. Proc. R. Soc. A311, 591.

Thomas, L. and NorTon, R B. (1967), Absorption of solar $x$ rays and density changes between 140 and 160 kilometers. J. geophys. Res. 72, 5552.

Thomas, L., Venables, F. H. and Williams, K. M. (1965). Measurements of solar $x$-ray fluxes by the U.S. Naval Research Laboratory satellite 1964-01-D. Planet. Space Sci. 13, 807.

Venkateswaran, S. V., Moore, J. G. and Krueger, A. J. (1961). Determination of the vertical distribution of ozone by satellite photometry. J. geopliys. Res. 66, 1751.

WATANABE, K. (1958). UV absorption processes in the upper atmosphere. Advan. Geophys. 5, 153.

WeEks, L. H. and SMrTH, L. G. (1968). Molecular oxygen concentrations in the upper atmosphere by absorption spectroscopy. J. geophys. Res. $\mathbf{7 3}, 4835$.

Wildman, P. J. L., Kerby, M. K. and ShaW, M. S. (1969). Molecular oxygen measurements from 100 to $150 \mathrm{~km}$ at Woomera, Australia. J. atmos. terr. Phys. 31, 951. 
APPENDIX

Several of the coefficients discussed in the text are presented in this Appendix.

where

$$
\begin{gathered}
\Delta \alpha_{i}=\frac{\sum_{j=K_{1}}^{K_{2}}\left[\frac{\exp \left(-\beta_{0 i}\left(z_{j}-z_{i}\right)\right)}{\left\{\bar{z}_{i}^{1}\right\}}-\frac{\left(z_{j}-z_{i}\right) \exp \left(-\beta_{0 i}\left(z_{j}-z_{i}\right)\right)}{\left\{\bar{z}_{i}^{2}\right\}}\right] \Delta N_{j}}{\frac{\left\{\bar{z}_{i}^{0}\right\}}{\left\{\bar{z}_{i}^{1}\right\}}-\frac{\left\{\bar{z}_{i}^{1}\right\}}{\left\{\bar{z}_{i}^{2}\right\}}} \\
\Delta \beta_{i}=\frac{1}{\alpha_{0 i}}\left\{\frac{\sum_{j=K_{1}}^{K_{2}}\left[\frac{\exp \left(-\beta_{0 i}\left(z_{j}-z_{i}\right)\right)}{\left\{\bar{z}_{i}^{0}\right\}}-\frac{\left(z_{j}-z_{i}\right) \exp \left(-\beta_{0 i}\left(z_{j}-z_{i}\right)\right)}{\left\{\bar{z}_{i}^{1}\right\}}\right] \Delta N_{j}}{\frac{\left\{\bar{z}_{i}^{2}\right\}}{\left\{\bar{z}_{i}^{1}\right\}}-\frac{\left\{\bar{z}_{i}^{1}\right\}}{\left\{\bar{z}_{i}^{0}\right\}}}\right\}
\end{gathered}
$$

where

$$
\begin{gathered}
\left\{\bar{z}_{i}^{0}\right\}=\sum_{j=K_{1}}^{K_{1}} \exp \left(-2 \beta_{0 i}\left(z_{j}-z_{i}\right)\right) \\
\left\{\bar{z}_{i}^{1}\right\}=\sum_{j=K_{1}}^{K_{2}}\left(z_{j}-z_{i}\right) \exp \left(-2 \beta_{0 i}\left(z_{j}-z_{i}\right)\right) \\
\left\{\bar{z}_{i}^{2}\right\}=\sum_{j=K_{i}}^{K_{2}}\left(z_{j}-z_{i}\right)^{2} \exp \left(-2 \beta_{0 i}\left(z_{j}-z_{i}\right)\right) \\
\overline{\left\langle\Delta \alpha_{i}\right\rangle}=\frac{X_{i}}{F_{\infty}^{1 / 2}}
\end{gathered}
$$

$$
X_{i}=\left[\sum_{j=K_{2}}^{K_{2}}\left\{\frac{\left[\frac{\exp \left(-\beta_{0 i}\left(z_{j}-z_{i}\right)\right)}{\left\{\bar{z}_{i}^{1}\right\}}-\frac{\left(z_{j}-z_{i}\right) \exp \left(-\beta_{0 i}\left(z_{j}-z_{i}\right)\right)}{\left\{\bar{z}_{i}^{2}\right\}}\right]_{\sigma}\left(\frac{1}{F_{0 j}}\right)^{1 / 2}}{\left\{\bar{z}_{i}^{0}\right\}}\right]^{2}-\frac{\left\{\bar{z}_{i}^{1}\right\}}{\left\{\bar{z}_{i}^{2}\right\}}\right]^{1 / 2}
$$

and

where

$$
\overline{\left\langle\Delta \beta_{i}\right\rangle}=\frac{Y_{i}}{F_{\infty}^{1 / 2}}
$$

$$
\begin{array}{r}
Y_{i}=\left[\frac{1}{\alpha_{0 i}} \sum_{j=K, 1}^{K_{2}}\left\{\frac{\left[\frac{\exp \left(-\beta_{0 i}\left(z_{j}-z_{i}\right)\right)}{\left\{\bar{z}_{i}^{0}\right\}}-\frac{\left(z_{j}-z_{i}\right) \exp \left(-\beta_{0 i}\left(z_{j}-z_{i}\right)\right)}{\left\{\bar{z}_{i}^{1}\right\}}\right] \frac{1}{\sigma}\left(\frac{F_{\infty}}{F_{0 j}}\right)^{1 / 2}}{\left.\frac{\left\{\bar{z}_{i}^{2}\right\}}{\left\{\bar{z}_{i}^{1}\right\}}-\frac{\left\{\bar{z}_{i}^{1}\right\}}{\left\{\bar{z}_{i}^{0}\right\}}\right]^{2}}\right]^{1 / 2}\right. \\
R_{i}=\beta_{0 i} \varepsilon^{-1 / 2}\left\{\left(1+\frac{1}{2} \underline{\varepsilon}-\frac{1}{4 \beta_{0 i} \varepsilon}\right)\left(\frac{\pi}{\beta_{0 i}}\right)^{1 / 2}\left[\operatorname{erf}\left(\left(\beta_{0 i} \gamma\right)^{1 / 2}\right)-\operatorname{erf}\left(\left(\beta_{0 i} \Delta\right)^{1 / 2}\right)\right] \exp \left(\beta_{0} \Delta\right)\right. \\
\left.+\frac{1}{2 \beta_{0 i} \varepsilon}\left[\gamma^{1 / 2} \exp \left(-\beta_{0 i} \eta\right)-\Delta^{1 / 2}\right]\right\}
\end{array}
$$

where $\Delta=r_{i}-r_{i}$

$$
\begin{aligned}
\gamma & =r_{i+1}-r_{l} \\
\varepsilon & =r_{i}+r_{i} \\
\eta & =r_{i+1}-r_{i}
\end{aligned}
$$




$$
\begin{aligned}
& S_{i}=\left(\frac{\pi}{\beta_{0 i}}\right)^{1 / 2}\left[\operatorname{erf}\left(\left(\beta_{0 i} \gamma\right)^{1 / 2}\right)-\operatorname{erf}\left(\left(\beta_{0 i} \Delta\right)^{1 / 2}\right)\right]\left[\alpha_{0 i} \varepsilon^{-1 / 2}\left(1+\frac{1}{2} \Delta \varepsilon^{-1}-\left(4 \beta_{0 i} \varepsilon\right)^{-1}\right)\right. \\
& \left.-\alpha_{0 i} \beta_{0 i} \varepsilon^{-1 / 2}\left(\left(4 \beta_{0 i}\right)^{-1}\left(2+\Delta \varepsilon^{-1}\right)-\frac{1}{2}\left(2 \Delta+\Delta^{2} \varepsilon^{-1}\right)-\frac{3}{8} \beta_{0 i}{ }^{-2} \varepsilon^{-1}\right)\right] \\
& . \exp \left(\beta_{0 i} \Delta\right)+\left(2 \beta_{0 i}\right)^{-1}\left[\gamma^{1 / 2} \exp \left(-\beta_{0 i} \eta\right)-\Delta^{1 / 2}\right] \\
& \text {. }\left[\alpha_{0 i} \varepsilon^{-3 / 2}+\alpha_{0 i} \beta_{0 i} \varepsilon^{-1 / 2}\left(2+2 \Delta \varepsilon^{-1}\right)\right] \\
& -\alpha_{0 i} \beta_{0 i}\left(4 \beta_{0 i}^{2} \varepsilon^{3 / 2}\right)^{-1}\left[\gamma^{1 / 2}\left(2 \beta_{0 i} \gamma+3\right) \exp \left(-\beta_{0 i} \eta\right)-\Delta^{1 / 2}\left(2 \beta_{0 i} \Delta+3\right)\right] \\
& Q_{j}=\left[\left(\frac{P}{K}\left(z^{2}\right)-\frac{L(\bar{z})}{K}\right)+\frac{L M}{K} z_{j}-\frac{P M}{K} z_{j}^{2}\right] \\
& Q_{j}^{*}=\left[\left(\frac{L^{2}}{P K}(z)-\frac{(\bar{z})}{P}-\frac{L}{K} \overline{\left(z^{2}\right)}\right)+\left(\frac{M}{P}-\frac{L^{2} M}{P K}\right) z_{j}+\frac{L M}{K} z_{j}^{2}\right] \\
& L=\left(M\left(\overline{z^{3}}\right)-(\bar{z})\left(\overline{z^{2}}\right)\right) \\
& P=\left(M\left(\overline{z^{2}}\right)-(\bar{z})(\bar{z})\right)
\end{aligned}
$$

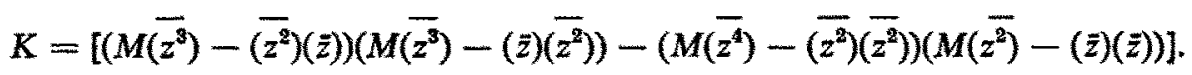

